\title{
Políticas públicas de protección del agua en el departamento del Huila ${ }^{1}$
}

\section{Public policy water protection in the department of Huila}

\author{
Germán Alfonso López Daza \\ Doctor en Derecho Constitucional- Université de Paris II (Panthéon - Assas) \\ Profesor Universidad Surcolombiana \\ germanlo@usco.edu.co \\ Carlos Fernando Gómez García \\ Candidato a Magíster en Derecho -Universidad Santo Tomas \\ carlos.garcía@usco.edu.co
}

Recibido: 03/02/2014 Aprobado: 01/06/2014

\section{RESUMEN}

El agua, como elemento indispensable para la existencia de los seres vivos, es actualmente, centro de atención por parte de la comunidad internacional, principalmente por el desmesurado aumento de la población humana y el gran desarrollo de ciertos países que cada vez requieren de más agua para sostener su población.

El paulatino agotamiento de los recursos naturales en los países industrializados ha puesto en la mira las grandes riquezas hidrográficas de los países en vía de desarrollo como Colombia. La compra masiva de tierras fértiles y nacimientos de agua por parte de multinacionales ha generado una discusión consistente en la protección de las riquezas fluviales mediante normativas que permitan a los países pobres asegurar su subsistencia.

El presente escrito, tiene como objetivo determinar el desarrollo de las políticas públicas nacionales, y del departamento del Huila, tendientes a garantizar el acceso y conservación del agua.

\section{PALABRAS CLAVE}

Agua, derecho fundamental, políticas, normativas, acceso.

\section{ABSTRACT}

Water, as indispensable to the existence of living beings member, is currently the center of attention from the international community, mainly the excessive increase in human population and the great development of certain countries, which increasingly require more water to sustain its population.

The gradual depletion of natural resources in developed countries has been targeted the great wealth of developing countries like Colombia. The massive buying fertile land and water a source by multinational has

1.- El presente artículo, constituye uno de los resultados de la investigación denominada: "Políticas públicas de protección del agua en el departamento del Huila", realizada en el año 2014 por el Semillero de Investigación Scholla adscrito al Grupo de Investigación Nuevas Visiones del Derecho, integrado por los estudiantes: Erika María Saldaña Hernández, Andrea Del Pilar Penagos Vera, Reinel Camilo Claros León, Luisa Fernanda Morales Zambrano y Jaime Andrés Montano Osorio. 
generated consistent in protecting river wealth through policy discussion to enable poor countries to secure their livelihood.

This project aims to determine the development of public policy national, and the Huila department aimed at ensuring access and conservation of water.

\section{KEYWORDS}

Water, fundamental law, policies, standards, access.

\section{INTRODUCCIÓN}

El derecho al agua se debe garantizar no sólo en condiciones de calidad, accesibilidad y disponibilidad $^{2}$, sino que debe tratarse como un bien social y cultural; lo que quiere decir, que el ejercicio de este derecho debe ser de tal forma que sea sostenible tanto para las generaciones actuales como para las futuras; por tanto surge encabeza de los Estados, la implementación de medidas y políticas adecuadas tendientes a garantizar la eficacia de los derechos y libertades, con el fin de suplir las necesidades alimenticias, agrícolas y tecnológicas que se derivan de ella.

A los gobiernos les corresponde administrar eficientemente este recurso hídrico y garantizarle este derecho a toda la población, para tal efecto, es obligación de los entes del orden departamental, desarrollar los Planes Departamentales para el Manejo Empresarial de los Servicios de Agua y Saneamiento (PDA), como estrategias de planeación y coordinación interinstitucional, formuladas y ejecutadas con el objeto de lograr la armonización integral de los recursos y la implementación de esquemas eficientes y sostenibles en la prestación de los servicios públicos de Agua Potable y Saneamiento Básico, en armonía con los lineamientos y políticas establecidas en el orden nacional.

En ese orden de ideas surge el presente proyecto de investigación, que planteó como problema de investigación: ¿Cómo ha sido la implementación y desarrollo de las Políticas Públicas en torno a la protección y al acceso del derecho al agua en el Departamento del Huila? Interrogante que surgió motivado por las múltiples intervenciones indiscriminadas al recurso hídrico por parte de multinacionales que buscan explotar el agua con fines económicos, sin tener en consideración las implicaciones ambientales; para esto se fijó como objetivo general determinar y analizar la implementación y desarrollo de las Políticas Públicas en torno a la protección y al acceso del derecho al agua en el Departamento del Huila.

De igual manera, como objetivos específicos se planteó, en primer lugar, identificar y analizar la normativa nacional y departamental tendiente a garantizar el acceso al agua potable y a conservar este recurso hídrico, seguidamente, consultar y examinar si las políticas públicas del agua en el orden departamental, cumplen con los parámetros establecidos a nivel nacional y finalmente, determinar si las políticas públicas en torno al agua cuentan con los componentes mínimos que garantizan su efectividad en el Departamento del Huila.

Por lo anteriormente expuesto, en el presente escrito se desarrollarán los siguientes aspectos: i). Se expondrá el análisis de los tratados y documentos internacionales que se relacionan con el derecho al agua; ii). Se analizará el agua como derecho fundamental desde la perspectiva nacional; luego, iii). Se explicará el tratamiento que la jurisprudencia de la Corte Constitucional le ha dado al recurso hídrico; seguidamente; iv). Se presentará el Plan Departamental de Agua y Saneamiento para el

2.-Para mayor profundización de estos conceptos, véase la Sentencia T-888 de 2008. 
Manejo Empresarial de los Servicios de Acueducto del Departamento del Huila, y finalmente; v). Se plantearán las conclusiones.

\section{METODOLOGÍA}

\section{a) Enfoque de la investigación: Cualitativo}

Teniendo en cuenta la problemática a abordar, el enfoque epistemológico más conveniente es el cualitativo, puesto que pretende estudiar las características del objeto a investigar: Normativa internacional y Nacional, Políticas Públicas Nacionales y Departamentales e igualmente la Jurisprudencia de la Corte Constitucional en torno a a la protección y al acceso del derecho al agua.

\section{b) Tipo de investigación: Descriptiva-Explicativa} Teniendo en cuenta la naturaleza de la investigación, se implementó una investigación descriptivaexplicativa, estudiando las propiedades del fenómeno a investigar: Políticas Públicas en torno a la protección y al acceso del derecho al agua en el Departamento del Huila.

Para lograr lo anterior, se desarrollaron los siguientes procedimientos:

- Inicialmente, se analizó el contexto internacional con el fin de identificar la normativa que protege el derecho al agua.

- Seguidamente, se estudió el desarrollo de las políticas públicas nacionales y del departamento del Huila en torno al agua.

- Posteriormente, se hizo una revisión de las sentencias proferidas por la Corte Constitucional, seleccionando aquellas que ponen de presente la protección y la accesibilidad al agua.

- Luego, se realizó un análisis individual de cada fallo analizando las implicaciones políticas y jurídicas.

- Finalmente, se identificó las tendencias actuales del derecho tendientes a la protección de este derecho.

\section{AVANCES, RESULTADOS, DISCUSIONES}

\subsection{Normativa internacional del derecho al agua}

El derecho humano al agua es el derecho de todos a disponer de agua suficiente, salubre, aceptable, accesible y asequible para el uso personal y doméstico (U.N. Doc. HRI/GEN/1/Rev.7 at 117, 2002).

Este derecho, ha sido acogido por un gran número de documentos internacionales, que buscan reconocerlo expresamente, como un derecho humano y fundamental para la realización de todos los demás derechos humanos, así se encuentran:

a) La Declaración Universal de los Derechos Humanos

b) Las reglas mínimas para el tratamiento de los reclusos

c) La Declaración de los Derechos del Niño

d) El Pacto Internacional de Derechos Civiles y Políticos

e) La Declaración Universal sobre la Erradicación del Hambre y la Malnutrición

f) La Convención sobre la eliminación de todas las formas de discriminación contra la mujer

g) Los Principios Rectores de los desplazamientos internos

h) La Observación general $N^{0} 15$ del Comité de Derechos Económicos, Sociales y Culturales

i) La Convención sobre los derechos de las personas con discapacidad

j) Los Objetivos de Desarrollo del Milenio

En el presente siglo, el acceso al agua ha sido reconocido como fundamental tanto por organismos internacionales, como por el Comité de Derechos Económicos, Sociales y Culturales en su Observación General No. 15 definiéndolo como "el derecho de todos de disponer de agua suficiente, salubre, aceptable, accesible y asequible para el uso personal o doméstico" (ONU, 2002).

De esta manera, el Derecho al agua es un derecho fundamental de todos los seres humanos, para lograr 
la efectividad de los demás derechos humanos. Por un lado, su aplicación, permite conseguir alternativas para combatir la problemática actual de la pobreza en el mundo; y a su vez, su reconocimiento, marca un paso elemental para derribar la desigualdad existente en la sociedad a la hora de acceder a este derecho.

De igual forma, gracias a la consagración de este derecho en las diversas convenciones, declaraciones y observaciones internacionales, se han preestablecido normas, con el fin de fijar planes para desafiar los problemas hídricos crecientes y sus posibles soluciones. Cabe resaltar, que es de vital importancia, la implementación de esta normatividad, para mejorar la calidad de vida y la salud de las personas, incluyendo su supervivencia, pues es claro, que todo esto depende de un acceso correcto y apropiado al agua.

Un tema interrelacionado con el derecho al agua, es el derecho al acceso a los servicios básicos de agua y saneamiento, que se ha considerado como un derecho fundamental, por tal razón, los Estados tienen la obligación de suministrar estos servicios a la población en general, sin discriminación alguna, y sin eximirse igualmente de este deber bajo la justificación de falta de recursos.

Finalmente es posible decir, que la necesidad de reconocer el derecho al agua como derecho en el marco de la normatividad internacional, es con el fin de brindarle el carácter de "bien común público" y prevenirlo de la categoría de los "bienes y servicios mercantiles", puesto que es un recurso vital, esencial e insustituible no solo para las actividades humanas, sino también para otras actividades de índole económica.

\subsection{El derecho fundamental al agua en Colombia}

Colombia, ha indicado que el agua es derecho fundamental, así mediante sentencia T-740/11, la Corte Constitucional como órgano encargado de la salva guarda de la Constitución y por ende la protección de los derechos de todos los ciudadanos, le concede una "doble connotación pues se erige como un derecho fundamental y como un servicio público, por lo tanto, estableciendo para el Estado la obligación de organizar, dirigir, reglamentar y garantizar su prestación de conformidad con los principios de eficiencia, universalidad y solidaridad", del que también hace parte los tratados descritos en el acápite anterior, de acuerdo con el bloque de constitucionalidad (Art. 93 C.P., 1991).

Dicho reconocimiento se hace debido a la importancia del líquido para la vida de los seres humanos, ya que se constituye como elemento esencial donde se da la vida y sin el cual no existiría, como lo constata la Organización Mundial de la Salud que a inicios del presente siglo reportó un informe en el que las cifras mostraban que 1.100 millones de personas (el $80 \%$ de ellas residentes en zonas rurales) carecían de un abastecimiento de agua capaz de suministrar por lo menos 20 litros diarios de agua potable por persona y se estimó que 2.400 millones no tenían acceso a servicios de saneamiento (O.M.S.), a causa de esto y demás problemas que aquejan a la humanidad a los cuales se busca hacer frente, se crearon los Objetivos de Desarrollo del Milenio (ODM) entre los cuales esta reducir a la mitad la proporción de personas sin acceso al agua potable mucho antes de la fecha límite de 2015. 


\begin{tabular}{|c|c|}
\hline OBJETIVO DE DESARROLLO DEL MILENIO & RELACIÓN CONELAGUA(ONU) \\
\hline Erradicar la Pobreza Extrema y el Hambre & $\begin{array}{l}\text { El acceso a agua para uso doméstico y productivo como } \\
\text { agricultura, industria y otras actividades económicas, } \\
\text { influyen directamente sobre la pobreza y la seguridad } \\
\text { alimentaria. }\end{array}$ \\
\hline Educación Universal & $\begin{array}{l}\text { La magnitud de acontecimientos catastróficos y cada } \\
\text { vez más recurrentes como las sequías, interrumpen el } \\
\text { proceso educativo. }\end{array}$ \\
\hline Igualdad entre Géneros & $\begin{array}{l}\text { El acceso al agua, en particular bajo condiciones de } \\
\text { escasos recursos, tiene importantes implicaciones de } \\
\text { género que afectan al capital social y económico de las } \\
\text { mujeres en términos de liderazgo, ingresos y } \\
\text { oportunidades de relacionarse. }\end{array}$ \\
\hline Reducir la Mortalidad de los Niños & \multirow{2}{*}{$\begin{array}{l}\text { Unos programas de gestión de los recursos hídricos } \\
\text { equitativos y fiables reducen la vulnerabilidad de los } \\
\text { más pobres frente a los imprevistos, lo que además les } \\
\text { proporciona unos medios de vida más seguros y } \\
\text { rentables de para el cuidado de sus hijos. }\end{array}$} \\
\hline Mejorar la Salud Materna & \\
\hline Combatir elVIH/Sida & $\begin{array}{l}\text { El acceso a una fuente de agua mejorada y a sistemas } \\
\text { de tratamiento de las aguas residuales en los } \\
\text { asentamientos humanos reduce la transmisión de los } \\
\text { riesgos ligados a enfermedades transmitidas por } \\
\text { mosquitos como la malaria yla fiebre del dengue. }\end{array}$ \\
\hline Sostenibilidad del Medio Ambiente & $\begin{array}{l}\text { Un tratamiento adecuado de las aguas residuales } \\
\text { contribuye a reducir la presión sobre los recursos de } \\
\text { agua potable, ayudando a proteger la salud humana y } \\
\text { del medio ambiente. }\end{array}$ \\
\hline Fomentar una Asociación Mundial & $\begin{array}{l}\text { La escasez de agua requiere reforzar cada vez más la } \\
\text { cooperación internacional en el campo de las } \\
\text { tecnologías para aumentar la productividad y las } \\
\text { oportunidades de financiación de los recursos hídricos } \\
\text { y un entorno mejorado para compartir los beneficios de } \\
\text { la gestión de la escasez del agua. }\end{array}$ \\
\hline
\end{tabular}

La comunidad internacional a través de sus organizaciones ha buscado la realización de estos objetivos y específicamente el del agua potable, con el que ha cumplido, como lo demuestra el informe que presentó la OMS el día 6 de marzo del año inmediatamente anterior (OMS, 2012), al respecto:

Entre 1990 y 2010, más de 2.000 millones de personas obtuvieron acceso a fuentes mejoradas de agua potable, como por ejemplo suministro de agua por medio de tuberías y pozos.

A finales de 2010 un $89 \%$ de la población mundial, o 6.100 millones de personas, utilizaban fuentes mejoradas de agua potable. Se trata de un $1 \%$ más que la cifra que figuraba en la meta de los ODM, un $88 \%$.

Sin embargo aún no es posible declarar victoria, ya que por lo menos el $11 \%$ de la población mundial -783 millones de personas - no tiene acceso aún al agua potable, y miles de millones no reciben todavía servicios de saneamiento (A.S.S., 2012). 
Lo anterior demuestra, que a pesar de que nuestra estirpe ha avanzado aún dependemos de este recurso de manera permanente e irreemplazable y a pesar de que nuestra tecnología ha evolucionado a tal punto de alcanzar altos niveles, aún en distintas partes del mundo mueren personas por deshidratación y consumo de agua no potable.

Por tal razón, es de gran importancia que las naciones se unan en la protección de sus fuentes hídricas que resultan esenciales para su desarrollo social y económico, y que representa el $0,025 \%$ de agua del planeta consumible para el ser humano (Informe geo america latina y el caribe - perspectiva del medio ambiente, 2003).

En Colombia, la entidad pública de orden nacional encargada por parte del Estado para que sirva de rector de la gestión del medio ambiente y de los recursos naturales renovables, encargado de impulsar una relación de respeto y armonía del hombre con la naturaleza y de definir, las políticas y regulaciones a las que se sujetarán la recuperación, conservación, protección, ordenamiento, manejo, uso y aprovechamiento de los recursos naturales renovables y el medio ambiente de la Nación, a fin de asegurar el desarrollo sostenible, es el Ministerio de Medio Ambiente creado en 1993 junto con el Sistema Nacional Ambiental (SINA) como conjunto de orientaciones, normas, actividades, recursos, programas e instituciones que permiten la puesta en marcha de los principios generales ambientales. (Ley 99/1993).

Es así, como por medio de estas diversas instituciones tienen la función de proteger los recursos naturales que se constituyen como una de las mayores riquezas de nuestro país, su presencia se extiende a los otros entes territoriales con el fin de ejercer su cometido junto a la comunidad, permitiendo tener una cobertura que acoja en su totalidad a los habitantes residentes en todo el territorio nacional.

La garantía de un mínimo vital para aquellas comunidades débiles y necesitadas del país se establece como una garantía de aquel derecho fundamental a ese líquido que representa las dos terceras partes del peso del cuerpo humano (Goldman, 2013), y que por consiguiente es necesario para la vida y el mejoramiento de las condiciones de salud, economía y desarrollo de las familias.

A nivel nacional se han expedido decretos y leyes que tienden a la protección del agua en nuestro territorio, así se encuentran:

Decreto 1594 de 1984, consagra los criterios de calidad que deben alcanzar las fuentes de agua para posibilitar los diferentes usos; en él se indican los criterios de calidad como guías para ser utilizadas para el ordenamiento, asignación de usos al recurso y determinación de las características del agua para cada uso.

Establece que los usos del agua serán: consumo humano y doméstico, preservación de flora y fauna, agrícola, pecuario, recreativo, industrial y transporte así como que en los sitios donde se asignen usos múltiples, los criterios de calidad para la destinación del recurso, corresponderán a los valores más restrictivos de cada referencia.

Ley 142 de 1994, determina como un derecho tanto de la Empresa de Servicio Público (ESP) como del usuario, la medición de los consumos de agua, indicando que éste debe ser el elemento principal del precio que se cobre al suscriptor o usuario en la tarifa.

También exige a todas las entidades prestadoras de servicios de acueducto y riego adelantar programas para instalar medidores de consumo a todos los usuarios cuyos costos pueden ser financiados por la ESP.

Decreto 475 de 1998, prescribe que el agua para consumo humano debe cumplir los requisitos de calidad microbiológica, organoléptica y fisicoquímica. De igual manera, el RAS indica que las corrientes hídricas superficiales están sometidas a contaminación, por lo cual sólo pueden considerarse como 
fuente de agua "potable", una vez se tomen medidas para la protección de la calidad del agua, como la vigilancia de la cuenca, aislamiento de las rondas hídricas e instalación de plantas de tratamiento de agua.

Decreto 302 de 2000, indica que los contratos de prestación del servicio pueden exigir a los suscriptores o usuarios la adquisición, instalación, mantenimiento y reparación de los instrumentos de medición de los consumos de agua cuando se establezca que el funcionamiento no permite determinarlos adecuadamente.

Es importante traer a colación la Resolución 1096 de 2000 (RAS) pues sujeta los lineamientos para definir los niveles de tratamiento del agua para "consumo humano", en función de la calidad de la fuente, que va desde aceptable a muy deficiente, de acuerdo con su grado de contaminación.

Decreto 1575 de 2007, incluye la elaboración de un mapa de riesgo como el "instrumento que permite definir las acciones de vigilancia y control del riesgo asociado a las condiciones de calidad de las cuencas abastecedoras de los sistemas de suministro de agua para consumo humano.

Resolución Numero 2115 Junio 22 de 2007, introduce uno de los cambios más notorios en la reglamentación de los indicadores "Índice de Riesgo de la Calidad de Agua para Consumo Humano IRCA" y el "Índice de Riesgo por Abastecimiento de Agua para Consumo Humano - IRABA", aspecto que facilita el seguimiento y el control de la calidad del agua. No obstante, resulta incomprensible que sea el mismo prestador el que realice el cálculo del IRCA y lo reporte al SUI - Sistema Único de Información, es decir que las acciones de vigilancia primaria recaen en el mismo prestador.

Por su parte, la Ley 1450 de 2011; en su artículo 250 señala que el Ministerio de Ambiente Vivienda y Desarrollo Territorial es el competente para la evaluación y viabilización de los proyectos del sector de agua potable y saneamiento básico que soliciten apoyo financiero de la Nación y sus entidades públicas descentralizadas a través del mecanismo que defina.

Los proyectos anteriormente nombrados involucran al el sector de agua potable y saneamiento básico financiados exclusivamente con recursos de las entidades territoriales en el marco de los Planes Departamentales para el manejo empresarial de los servicios de Agua y Saneamiento, serán evaluados y viabilizados a través de un mecanismo regional, conforme a la reglamentación que para el efecto expida el Gobierno Nacional.

Decreto Número 1873 de 2012, que reglamenta el artículo 250 de la Ley 1450 de 2011, se crea el Mecanismo Departamental de Evaluación, Viabilización y Aprobación de Proyectos del Sector de Agua Potable y Saneamiento Básico priorizados en el marco de los Planes Departamentales de Agua y de los programas regionales y/o departamentales que implemente el Ministerio de Vivienda, Ciudad y Territorio, se establecen sus requisitos y se dictan otras disposiciones. 


\section{EL AGUA EN LA JURISPRUDENCIA DE LA CORTE CONSTITUCIONAL: EL AGUA COMO SERVICIO PÚBLICO DOMICILIARIO}

\begin{tabular}{|c|c|c|}
\hline \multirow{3}{*}{ AÑO 1992} & T-406 & $\begin{array}{l}\text { El derecho a la salubridad pública y al servicio de alcantarillado público, } \\
\text { se consideran derechos susceptibles de protección por medio de la } \\
\text { acción de tutela, en el entendido de que si no es debidamente garantizado } \\
\text { afectará de manera evidente derechos y principios constitucionales } \\
\text { fundamentales, como la dignidad humana, la vida y los derechos de los } \\
\text { disminuidos. }\end{array}$ \\
\hline & T-570 & $\begin{array}{l}\text { Cuando el Estado no se encuentra en condiciones de prestar } \\
\text { debidamente un servicio público que es esencial para la población, debe } \\
\text { crear las condiciones para que o los particulares o las comunidades } \\
\text { organizadas puedan prestarlos directamente. }\end{array}$ \\
\hline & T-578 & $\begin{array}{l}\text { Los servicios públicos "domiciliarios" son aquellos que se prestan a través } \\
\text { del sistema de redes físicas o humanas con puntos terminales en las } \\
\text { viviendas o sitios de trabajo de los usuarios y cumplen la finalidad } \\
\text { específica de satisfacer las necesidades esenciales de las personas. }\end{array}$ \\
\hline AÑO 1995 & T- 413 & $\begin{array}{l}\text { El derecho al agua, para el uso de las personas, en cuanto contribuye a la } \\
\text { salud, a la salubridad pública, y, en últimas, a la vida, si es un derecho } \\
\text { fundamental y que, por el contrario, no lo es cuando se destina a la } \\
\text { explotación agropecuaria o a un terreno deshabitado. }\end{array}$ \\
\hline AÑO 1998 & T-578 & $\begin{array}{l}\text { Los servicios públicos se erigen como instrumentos que le permiten al } \\
\text { Estado alcanzar el ideal de justicia social y promover condiciones de } \\
\text { igualdad real y efectiva, bien sea que los preste directa o indirectamente, } \\
\text { o que autorice a los particulares para hacerlo, en todo caso siempre serán } \\
\text { su responsabilidad, la cual deberá cumplir de acuerdo con las } \\
\text { disposiciones de la ley que rija su prestación, tal como lo dispone el } \\
\text { artículo } 365 \text { de la Constitución. }\end{array}$ \\
\hline AÑO 2007 & $\mathrm{~T}-270$ & $\begin{array}{l}\text { Se pronuncian respecto de la suspensión de los servicios públicos } \\
\text { domiciliarios por parte de las empresas prestadoras en sujetos de } \\
\text { especial protección. }\end{array}$ \\
\hline AÑO 2010 & T- 418 & $\begin{array}{l}\text { La protección y garantía adecuada de las dimensiones prestacionales de } \\
\text { los derechos fundamentales constitucionales, bien sean de libertad, bien } \\
\text { sean sociales, depende en buena parte de las políticas públicas que, } \\
\text { dentro del orden constitucional vigente, sean diseñadas, elaboradas, } \\
\text { implementadas, evaluadas y controladas, en un contexto de democracia } \\
\text { participativa. }\end{array}$ \\
\hline \multirow[t]{2}{*}{ AÑO 2011} & T-055 & $\begin{array}{l}\text { A través del derecho al agua potable y su acceso efectivo en la prestación } \\
\text { del servicio de acueducto se está dando alcance a uno de los fines } \\
\text { esenciales del Estado como es la solución de las necesidades básicas } \\
\text { insatisfechas de las personas y la protección de otros derechos } \\
\text { fundamentales tan trascendentales como la vida en condiciones dignas y } \\
\text { la salud. }\end{array}$ \\
\hline & T-279 & $\begin{array}{l}\text { Hace mención a los deberes de las empresas prestadoras del servicio y } \\
\text { de los usuarios. }\end{array}$ \\
\hline
\end{tabular}




\begin{tabular}{|c|c|c|}
\hline \multirow[b]{2}{*}{ AÑO 2012} & T-385 & $\begin{array}{l}\text { Reitera que a pesar del incumplimiento de pago, las empresas de } \\
\text { servicios públicos domiciliarios no deben suspender los servicios } \\
\text { públicos cuando ello conduzca a una grave afectación de las condiciones } \\
\text { de vida del usuario, generándose un perjuicio irremediable. }\end{array}$ \\
\hline & T-752 & $\begin{array}{l}\text { En un Estado Social de Derecho como el nuestro, el suministro de los } \\
\text { servicios públicos, no puede depender de la mayor rentabilidad que la } \\
\text { prestación de los mismos genere, sino que debe obedecer a la } \\
\text { materialización de los principios y teleología recogidos en la carta política, } \\
\text { los cuales propenden por la igualdad real y efectiva y por el respeto de la } \\
\text { dignidad humana de todos los habitantes del territorio nacional. }\end{array}$ \\
\hline \multirow[b]{2}{*}{ AÑO 2013} & T-188 & $\begin{array}{l}\text { El agua en el ordenamiento jurídico colombiano tiene una doble } \\
\text { connotación pues se erige como un derecho fundamental y como un } \\
\text { servicio público. }\end{array}$ \\
\hline & T-273 & $\begin{array}{l}\text { El derecho a la continuidad en la prestación del servicio público } \\
\text { domiciliario de agua potable, cuando sus usuarios son sujetos de } \\
\text { especial protección constitucional, de conformidad con el ordenamiento } \\
\text { jurídico colombiano, amerita consideraciones superiores, por constituir el } \\
\text { acceso al agua potable un derecho fundamental, y por tratarse de un } \\
\text { servicio público, de manera que todas las personas deben poder acceder } \\
\text { al servicio de acueducto en condiciones de cantidad y calidad suficiente y } \\
\text { al Estado le corresponde organizar, dirigir, reglamentar y garantizar su } \\
\text { prestación de conformidad con los principios de eficiencia, universalidad } \\
\text { y solidaridad. }\end{array}$ \\
\hline AÑO 2014 & T-242 & $\begin{array}{l}\text { La suspensión de los servicios públicos domiciliarios es "un deber y un } \\
\text { derecho de las empresas prestadoras, el cual deben efectuar cuando } \\
\text { pasados dos periodos sucesivos de facturación el usuario no realice el } \\
\text { pago correspondiente; no obstante, esto no podrá tener lugar si: i). Se } \\
\text { violan las garantías al debido proceso; ii). Aun cuando se respete el } \\
\text { debido proceso pero se desconozcan derechos constitucionales de } \\
\text { sujetos que merecen una especial protección constitucional, se impida el } \\
\text { funcionamiento de hospitales u otros establecimientos igualmente } \\
\text { protegidos o se afecten gravemente las condiciones de vida de toda una } \\
\text { comunidad. }\end{array}$ \\
\hline
\end{tabular}

3. PROGRAMA AGUA Y SANEAMIENTO PARA LA PROSPERIDAD PLANES DEPARTAMENTALES DE AGUA Y SANEAMIENTO PARA EL MANEJO EMPRESARIAL DE LOS SERVICIOS DE ACUEDUCTO PAPPDA YEL PAP-PDADEL DEPARTAMENTO DEL HUILA

Los Programas Agua y Saneamiento para la Prosperidad - Planes Departamentales de Agua y Saneamiento para el manejo empresarial de los servicios de acueducto PAP-PDA y por lo tanto el PAP-PDAdel departamento del Huila han sido definidos por el documento CONPES 3463 de 2007, los Decretos 3200 de 2008 (el cual fue el regulador de los Planes Departamentales de Agua), 4214 de 2011 (este decreto modificó los Planes Departamentales de Agua y los adecuó a la política pública llamada prosperidad para todos del actual presidente Juan Manuel Santos) y 2246 de 2012 como un conjunto de estrategias de planeación y coordinación interinstitucional, formuladas y ejecutadas con el objeto de lograr la armonización integral de los recursos, y la implementación de esquemas eficientes y sostenibles en la prestación de los servicios públicos domiciliarios de 
agua potable y saneamiento básico.

Los PAP-PDA según las normas anteriormente mencionadas permitirán darle solución a las limitaciones que sufre la organización de la prestación del servicio de agua potable y saneamiento básico bajo una estructura ordenada, con visión integral y regional (una descentralización fuerte, para que cada Municipio analice su problemática y mediante estudios técnicos se encuentre la mejor forma de solucionarlo), mayor eficiencia y eficacia del Estado en sus acciones de apoyo al desarrollo del sector y de control, al tener como agentes coordinadores a los Departamentos.

Como parte de la política de puesta en marcha de los Planes Departamentales del Agua en el país, el Gobierno Nacional a través de el Ministerio de Vivienda, Ciudad y Territorio - MVCT- antiguamente Ministerio de Ambiente Vivienda y Desarrollo Territorial - MAVDT- emitió el Decreto 3200 de 2008 por el cual se dictan normas sobre los Planes Departamentales para el Manejo Empresarial de Agua y Saneamiento - PDA. Este Decreto fue modificado por el Decreto 4214 de 2011 y 2246 de 2012, los cuales reglamentan la Ley 1450 de 2011, los cuales estructuran los Planes Departamentales de Agua y los convierte en Programa Agua y Saneamiento para la Prosperidad Planes Departamentales de Agua y saneamiento para el manejo empresarial de los servicios de acueducto PAPPDA, de acuerdo a las características de la Ley 1450 de 2011, dicha norma es la que establece el plan de desarrollo nacional para los años comprendidos entre el 2010 y el 2014.

Los participantes en los planes departamentales de agua son:

i). El departamento, en nuestro caso sería el departamento del Huila.

ii). Los municipios y/o distritos, en el PAP- PDA del departamento del Huila hacen parte todos los municipios excepto el municipio de Elías el cual no firmó el convenio inter institucional, y la capital del departamento, el municipio de Neiva hace parte del PDA del departamento del Huila mediante Acuerdo 042 de 2008 pero como lo establece el artículo 7 del Decreto 3200 de 2008, los proyectos de inversión del Plan Departamental de Aguas, en esta capital, serán ejecutados por Empresas Públicas de Neiva E.S.P., como operador especializado de los servicios de acueducto, alcantarillado y asesor de la ciudad, siguiendo los lineamientos que al respecto defina el Viceministerio de Agua y Saneamiento.

iii). El Ministerio de Vivienda, Ciudad y Territorio MVCT.

iv). El Departamento Nacional de Planeación - DNP.

v). Las autoridades ambientales con jurisdicción en los municipios ubicados en el territorio del respectivo departamento. En este caso sería la Corporación Autónoma Regional del Alto Magdalena CAM.

Estos son los principales actores de Plan Departamental de Agua y Saneamiento Básico según el documento conpes 3463 de 2007 :

Estos entes están vinculados por convenios interinstitucionales, por lo tanto nace de la autonomía de la voluntad de los entes territoriales si hacen parte o no de los PDA, bajo los requisitos y en los términos que estableció el Decreto 3200 de 2008 en su artículo numeral 2, de los cuales el departamento del Huila suscribió cabalmente siguiendo esta cronología:

i). El 8 de Agosto de 2006 el departamento del Huila suscribió con el Ministerio de Ambiente Vivienda y Desarrollo Territorial - Dirección de Agua Potable y Saneamiento básico - el Acta de Compromiso mediante la cual se compromete a aunar esfuerzos para participar en la formulación e implementación del Plan Departamental de Agua y a vincularse se manera activa al mismo,

ii). En Mayo de 2007 la Gobernación del Huila suscribe con el Ministerio de Ambiente Vivienda y Desarrollo Territorial el Convenio de Asistencia técnica para la contratación de una firma consultora para que realice los estudios para el diagnostico y la estructuración, tendientes a la 
formulación del programa Plan Departamental de Agua y Saneamiento Básico en el departamento del Huila y la asistencia durante la fase de ejecución del contrato de consultoría.

iii). Mediante Ordenanza 031 del 21 de diciembre de 2007, la Asamblea Departamental del Huila otorgó facultades al Gobernador del Departamento para comprometer vigencias futuras de carácter excepcional con el fin de apalancar y soportar el plan de obras e inversiones resultantes de la estructura del plan departamental de Agua y Saneamiento.

iv). El 13 de Agosto de 2008 mediante Decreto 902 de 2008, la Gobernación del Huila adopto el Plan Departamental de Agua y Saneamiento Básico del departamento del Huila, y por lo tanto estableció la estructura operativa del mismo.

Los PAP- PDA tienen una estructura operativa que establece los lineamientos que se deben seguir y son los responsables que los mismos se cumplan cabalmente y esta estructura está compuesta por:

i). El Comité Directivo;

ii). El Gestor; en este caso mediante Decreto No. 962 del 27 de agosto de 2008 se estableció que el gestor del departamento del Huila es Aguas del Huila S.A. E.S.P., a excepción del municipio de Neiva cuyo gestor es Empresas públicas de Neiva E.S.P.

iii). La Gerencia Asesora, actualmente el PAP-PDA del departamento del Huila no han realizado esta contratación, por lo tanto esta función la suple Aguas de Huila, y

iv). El Esquema Fiduciario para el manejo de los recursos, el cual para el PAP-PDA del departamento del Huila es el Financiamiento de Inversiones en Agua - FIA

Los PAP-PDA están fijados para desarrollarse en 3 fases asi:

\section{Fase I-Diagnóstico}

Los diagnósticos departamentales que se realizarán en esta fase serán integrales en cuanto a los ámbitos que abarcarán (técnico, institucional, ambiental, financiero, social), y se llevarán a cabo con una visión regional, en la medida en que permitirán identificar potenciales esquemas regionales de prestación de los servicios para el aprovechamiento de economías de escala en la administración, operación e inversión, así como una óptima utilización del recurso hídrico en las regiones.

\section{Fasell-Estructuración}

El resultado de la Fase I se determina con el informe de la consultoría en la cual cada municipio debía elaborar una Plan Maestro de Acueducto y alcantarillado basado en su POT, PBOT o EOT dependiendo de la categoría del municipio, el cual le permitirá a la Gobernación, con el apoyo del Gobierno Nacional, definir la estructura final del Plan Departamental en aspectos técnicos, institucionales y financieros. La priorización que se establezca como resultado de las consultorías será un insumo para las Audiencias Públicas Consultivas, que se realizarán dentro del proceso de asignación de los aportes que hará la Nación como inversiones regionales del Plan Nacional de Desarrollo.

\section{Fase III - Implementación y Seguimiento}

La implementación del Plan Departamental de Agua y Saneamiento la liderará la Gobernación con el apoyo de una Gerencia Integral, la cual entre otras actividades, deberá ajustar, especificar y ejecutar el plan de choque de inversiones definido por la consultoría de diagnóstico, estructurar esquemas regionales para la vinculación de operadores especializados, apoyar la renegociación de contratos existentes ajustando las metas de acuerdo con los recursos adicionales aportados, el seguimiento a los contratos y el manejo financiero del Plan.

Según la información que reposa en las actas del Comités Directivo del PAP- PDA del departamento del Huila, se han priorizado 28 Planes Maestros de Acueducto y Alcantarillado, de los cuales su estado; 11 se han devuelto por el MVCT para ajustes, 13 están aprobados y en proceso de contratación los cuales corresponde a los municipios de: Baraya, Tello, Algeciras, Rivera, El Agrado, Pital, Villa Vieja, Altamira, Timana, Hobo, Guadalupe, Centro poblado 
la Victoria y el Centro poblado el Naranjal, y 4 ya se encuentra ejecutándose que corresponden a los municipios de Colombia, Tarqui, Paicol y Santa María.

Por lo anterior, se puede inferir que el PAP-PDA del Departamento del Huila se encuentra en la Fase 3 que corresponde a la implementación y seguimiento de los Planes Maestros, ya que de los 28 existentes; 13 esta aprobados, 4 ya están en ejecución y los otros 11 están siendo ajustado según los lineamientos establecidos por el Ministerio de Vivienda, Ciudad y Territorio - MVCT.

Cabe mencionar que en el último encuentro de la Federación Nacional de Departamentos que se realizó en la ciudad de Bogotá el pasado mes de Septiembre del año 2013 se hizo seguimiento a los PAP-PDAen general, y el PAP-PDA del Huila recibió una Felicitación por parte del el Ministerio de Vivienda, Ciudad y Territorio - MVCT, por la celeridad prestada en la viabilizacion de Planes Maestros en los últimos 6 meses bajo la administración de Carlos Mauricio Iriarte, siendo así uno de los PAP-PDA pilotos del país.

\section{CONCLUSIONES}

El derecho al agua, es la base fundamental de cada uno de los derechos humanos; por lo tanto, se han acogido con el paso de los años, diversos e innumerables instrumentos internacionales sobre dicho derecho. Estos, promueven e impulsan en su mayoría, el suministro y purificación de las aguas residuales, así como la completa satisfacción de las necesidades básicas del ser humano. Cabe resaltar, que el propósito primordial de estos mecanismos internacionales, es fijar mediante sus normativas, que el derecho al agua, es un derecho fundamental que debe ser de todos y para todos sin ninguna clase de discriminación.

El agua ha adquirido con el pasar del tiempo la connotación de derecho fundamental, que como fuente de vida le corresponde, por medio de instrumentos internacionales que han trascendido a nuestro ordenamiento interno, trayendo consigo fuertes críticas en lo que respecta a los tres aspectos que rodean su adecuada garantía (disponibilidad, accesibilidad y calidad), revelándonos una realidad que se configura en mucha ocasiones violatorias de las prerrogativas que la Corte como máxima autoridad a establecido en sus múltiples pronunciamientos que ostentan para este caso fuerza vinculante.

Surgiendo como consecuencia de la presencia de este tipo de problemáticas, una variedad de tentativas soluciones por parte de las autoridades estatales (ejecutivo y legislativo) con el fin de poner freno a las carencias de las que adolece la población en esta área específica; mediante la creación de organismos que tienen como centro de funcionamiento la protección de los recursos naturales, entre los que se encuentra el agua ocupando un lugar principal entre estos, además de la presentación de políticas y proyectos de actos legislativos que buscan proporcionar una mayor cobertura y protección a este recurso y por ende a los habitantes del territorio nacional como últimos destinatarios.

Desde el año 1992 el derecho a la salubridad pública y el servicio de alcantarillado público, se consideran derechos susceptibles de protección por medio de la acción de tutela, en el entendido de que si no es debidamente garantizado afectara de manera evidente derechos y principios constitucionales fundamentales, como la dignidad humana, la vida y los derechos de los disminuidos, así como también se contempla que el agua se considera derecho fundamental en el entendido de que su aprovechamiento está dirigido a la satisfacción de las necesidades básicas de las personas que hacen uso de ella y en cuanto contribuye a la salud, a la salubridad pública, y, en últimas, a la vida, pero que por el contrario, no se considera como derecho fundamental cuando se destina a la explotación agropecuaria o a un terreno deshabitado. Lo anterior teniendo como base fundamental el artículo 365 de nuestra carta, en donde se enuncia que los servicios públicos se erigen como instrumentos que le 
permiten al Estado alcanzar el ideal de justicia social y promover condiciones de igualdad real y efectiva, bien sea que los preste directa o indirectamente, 0 que autorice a los particulares para hacerlo.

Los PAP-PDAy por lo tanto el PAP-PDA del departamento del Huila, constituyen un gran avance respeto a la descentralización administrativa, ya que estas estrategias son desarrolladas básicamente por los municipios los cuales analizan sus problemáticas y proponen soluciones técnicas en un plan maestro, los departamentos solo son coordinadores.

Lo más importante de estas estrategias es que están visualizadas para mejorar las condiciones de vida de la mayoría de la población, tanto urbana como rural, siguiendo los principios de progresividad. Desafortunadamente el gobierno nacional y el documento CONPES, no incorporo en los PAP-PDA, la creación y/o remodelación de plantas de tratamiento de agua potable, por lo cual los PAP - PDA quedan técnicamente incompletos, porque proponen la infraestructura para proporcionar el vital liquido, pero en las urbes que requieren mayor grado de prestación o como se puede llegar a prestar el servicio en la zona rural, viendo que se necesitan obligatoriamente las plantas de tratamientos. Estas y la infraestructura que proponen los PAP-PDA son una pareja inseparable.

\section{REFERENCIAS BIBLIOGRÁFICAS}

- Dworkin, R. (2002). "Los derechos en serio" Barcelona Editorial: Ariel.

- García, M. E. (2007). Compendio de normas internacionales: derecho al agua.

- Rawls, J. (2004). El derecho de gentes y"Una revisión de la idea de la razón pública" Barcelona Editorial: Paidós.

- U.N. Doc. HRI/GEN/1/Rev.7 at 117 . (2002). Universidad de Minnesota.

- Univalle, Colciencias, Cinara. Aportes para la implementación de la política nacional en la gestión integral del recurso hídrico $(\mathrm{GIRH})$, Agua gran encuentro colombiano" cada gota tiene su historia.

\section{Páginas web}

- Convención Internacional sobre la Eliminación de todas las Formas de Discriminación Racial. (1965). Disponible en:

http://www2.ohchr.org/spanish/law/cerd.htm. Consultado: Marzo de 2013.

- Pacto Internacional de Derechos Civiles y Políticos. (1966). Disponible en:

http://www2.ohchr.org/spanish/law/ccpr.htm. Consultado mrzo de 2013

- Pacto Internacional de Derechos Económicos, Sociales y Culturales. (1966). Disponible en: http://www2.ohchr.org/spanish/law/cescr.htm. Consultado marzo d 2013.

- Declaración universal sobre la erradicación del hambre y la malnutrición. (1974). Dispnible en : http://cd3.uniandes.edu.co/sistema_derechos_h umanos/sistemas_principales/sistema_universal /documentos/tratados/declaracion_universal_so bre_la_erradicacion_del_hambre_y_la_malnutri cion. Consultado marzo de 2013.

- Reglas mínimas para el tratamiento de los reclusos. (1977). Disponible en:

http://www2.ohchr.org/spanish/law/reclusos.htm. Consultado. marzo de 2013.

- Convencion sobre la eliminacion de todas las formas de discriminacion contra la mujer. Disponible en:

http://www.un.org/womenwatch/daw/cedaw/text/ sconvention.htm. Consultado mayo de 2013.

- Convención sobre los Derechos del Niño. (1989). Disponible en: http://www.unicef.org/mexicol spanish/mx_Convencion_Derechos_es_final.pdf Consultado: marzo 2013.

- Convención sobre los Derechos del Niño. (1990). Disponible en: http://www2.ohchr.org/spanish/ law/crc.htm. Consultado en marzo de 2013.

- Revista Internacional de la Cruz Roja. (1998). Principio 18. [UN doc. E/CN.4/1998/53/Add.2, 11 de febrero de 1998. La Comisión de Derechos Humanos tomó nota de estos Principios Rectores.

- El derecho al agua. (2002). Artículos 11 y 12 del Pacto Internacional.

- Observación general $\mathrm{N}^{0}$ 15. (2002). Disponible en: http://www.escr-net.org/docs/i/428718. Consultado marzo de 2013. 
- Convención sobre los Derechos de las Personas con Discapacidad. (2006). Disponible en: http://es.wikisource.org/wiki/Convenci\%C3\%B3n _sobre_los_Derechos_de_las_Personas_con_D iscapacidad_y_su_Protocolo_Facultativo_de_la s_Naciones_Unidas. Consultado: marzo de 2013.

- 117, U. D. (2002). Observación general 15, Aplicación del Pacto Internacional de los Derechos Económicos, Sociales y Culturales, El derecho al agua (artículos 11 y 12 del Pacto), (29 período de sesiones 2002).

- Universidad de los Andes (2012). "El agua como derecho fundamental de los colombianos". Disponibleen:

http://congresovisible.org/agora/post/el-aguacomo-derecho-fundamental-de-los-colombianos/4007/. Consultado Octubre 2013.

- Declaracion Universal de los Derechos Humanos, Articulo 25. (1948). https://www.un.org les/documents/udhr/.

- Goldman L, Ausiello D, eds. Cecil Medicine. 24th ed. Philadelphia, Pa: Saunders Elsevier; (2011). Disponible en:

http://www.nlm.nih.gov/medlineplus/spanish/enc y/article/002471.htm. Consultado Octubre 2013

- Greenpeace Argentina (2003). "Informe geo américa latina y el caribe" perspectiva del medio ambiente. Disponible en:

http://www.greenpeace.org/argentina/es/campan as/contaminacion/agua/. Consultado octubre 2013.

\section{Referencias jurisprudenciales}

- C.Const, T-242/2013, Hernández.

- C.Const, T-273/2012, N.Pinilla.

- C.Const, T-055/2011, J.Palacio

- C.Const, T-188/2012, H.Sierra.

- C.Const, T-270/2007, J.Araujo.

- C.Const, T-279/2011, L.Vargas.

- C.Const, T-385/2011, N.Pinilla.

- C.Const, T-406/1992, C.Angarita.

- C.Const, T-413/1995, C.Angarita.

- C.Const, T-418/2010, M.Calle.

- C.Const, T-570/1992, J.Sanin.

- C.Const, T-578/1992, A.Martinez.
- C.Const, T-578/1998, F.Moron.

- C.Const, T-752/2011, J.Palacio

- CConst, T-740/2011, H. Sierra.

- Constitución Política de 1991

Referencias normativas

- Observación general 15, Aplicación del Pacto Internacional de los Derechos Económicos, Sociales y Culturales. (2002).

- Organización de las Naciones Unidas, Objetivos de Desarrollo del Milenio.

- Organización Mundial de la Salud O.M.S. (2012). "Progresos sobre el agua potable y saneamiento". Disponible en:

http://www.who.int/water_sanitation_health/monit oring/jmp2012/es/. Consultado Marzo 2013

- República de Colombia, Asamblea del Huila, Ordenanza 031 del 21 de Diciembre de 2007.

- L. $1151 / 2007$.

- L. $142 / 1994$.

- L. $1450 / 2011$.

- L. 19/1958.

- L.60/1993.

- L. 99/1993

- República de Colombia, Consejo Nacional de Política Económica y Social, Documento Conpes 3383 de 2005.

- República de Colombia, Consejo Nacional de Política Económica y Social, Documento Conpes 3463 de 2007.

- República de Colombia, Gobernación del Huila, Decreto 902 del 13 de Agosto de 2008.

- República de Colombia, Gobernación del Huila, Decreto 962 del 27 de Agosto de 2008.

- República de Colombia, Ministerio de Ambiente, Vivienda y Desarrollo Territorial, Decreto 3200 de 2008.

- República de Colombia, Ministerio de Ambiente, Vivienda y Desarrollo Territorial, Viceministerio de Agua y Saneamiento, Documento Plan Departamental para el Manejo Empresarial de los Servicios de Agua y Saneamiento del Huila.

- República de Colombia, Ministerio de Vivienda, Ciudad y Territorio, Decreto 2246 de 2012.

- República de Colombia, Ministerio de Vivienda, Ciudad y Territorio, Decreto 4214 de 2011. 